\title{
Reconstrução de Espectros de Raios X de Aceleradores Lineares Clínicos usando o Método de Recozimento Simulado Generalizado
}

\author{
Reconstruction of X-rays Spectra of Clinical Linear Accelerators using
} the Generalized Simulated Annealing Method

\author{
John Peter O. Manrique, Alessandro M. Costa \\ Faculdade de Filosofia, Ciências e Letras de Ribeirão Preto (USP), Ribeirão Preto (SP), Brasil
}

\begin{abstract}
Resumo
A distribuição espectral de raios $X$ de megavoltagem utilizados em departamentos de radioterapia é uma grandeza fundamental a partir da qual, em princípio, todas as informações requeridas relevantes para tratamentos de radioterapia podem ser determinadas. Para o calculo das doses administradas ao paciente que faz radioterapia se usam sistemas de planejamento de tratamento (TPS, do inglês Treatment Planning System), que fazem uso de algoritmos de convolução e superposição os quais requerem um conhecimento prévio do espectro de fluência de fótons para realizar o cálculo acurado das doses tridimensionais e assim assegurar uma melhor probabilidade de controle tumoral mantendo baixa a probabilidade de complicações do tecido normal. Neste trabalho foi obtido o espectro de fluência de fótons de raios $X$ de um acelerador linear clínico SIEMENS ONCOR de $6 \mathrm{MV}$, usando um método de caráter inverso para a reconstrução dos espectros, a partir das curvas de transmissão de fótons medidas para diferentes espessuras de alumínio; o método usado para a reconstrução dos espectros é uma técnica estocástica conhecida como recozimento simulado generalizado (GSA, do inglês Generalized Simulated Annealing), baseado no trabalho da estatística de quase equilíbrio de Tsallis. Para a validação dos espectros reconstruídos foram calculadas as curvas de porcentagem de dose em profundidade (PDD, do inglês Percentage Depth Dose) para a energia de $6 \mathrm{MV}$ do acelerador, usando simulação Monte Carlo com o código PENELOPE, e a partir da PDD se fez o cálculo o índice de qualidade do feixe TPR $20 / 10$.

Palavras-chave: radioterapia; recozimento simulado generalizado; Monte Carlo; espectrometria; fótons.
\end{abstract}

\begin{abstract}
The spectral distribution of megavoltage $X$-rays used in radiotherapy departments is a fundamental quantity from which, in principle, all relevant information required for radiotherapy treatments can be determined. To calculate the dose delivered to the patient who make radiation therapy, are used treatment planning systems (TPS), which make use of convolution and superposition algorithms and which requires prior knowledge of the photon fluence spectrum to perform the calculation of three-dimensional doses and thus ensure better accuracy in the tumor control probabilities preserving the normal tissue complication probabilities low. In this work we have obtained the photon fluence spectrum of $X$-ray of the SIEMENS ONCOR linear accelerator of $6 \mathrm{MV}$, using an character-inverse method to the reconstruction of the spectra of photons from transmission curves measured for different thicknesses of aluminum; the method used for reconstruction of the spectra is a stochastic technique known as generalized simulated annealing (GSA), based on the work of quasi-equilibrium statistic of Tsallis. For the validation of the reconstructed spectra we calculated the curve of percentage depth dose (PDD) for energy of $6 \mathrm{MV}$, using Monte Carlo simulation with PENELOPE code, and from the PDD then calculate the beam quality index TPR 20110 .
\end{abstract}

Keywords: radiotherapy; generalized simulated annealing; Monte Carlo; spectrometry; photons.

\section{Introdução}

A radioterapia e uma técnica de tratamento clinico que usa radiação ionizante de alta energia para o tratamento de pacientes com câncer ${ }^{1-4}$, este tipo de tratamento se baseia na entrega de doses de radiação suficientemente altas para inibir 0 crescimento e proliferação das células tumorais causando a morte destas; mas ao entregar altas doses de radiação se deve considerar preservar o tecido sadio circundante a zona tratada que em alguns pacientes pode comprometer órgãos vitais críticos afetando sua saúde. É por este motivo que agências internacionais que regulam $o$ uso de radiação ionizante, como a $\mathrm{ICRU}^{2}$, na sua publicação 62 , recomendam o uso de uma dose administrada ao paciente na faixa de $+7 \%$ e $-5 \%$ da dose prescrita pelo médico radioterapeuta.

Atualmente nos serviços de radioterapia se usam computadores de alta velocidade $e$ capacidade de processamento de dados, que tem instalados softwares especializados para o cálculo das doses tridimensionais no paciente, chamados sistemas de planejamento do tratamento ${ }^{4}$ (TPS, do inglês Treatment Planning System), estes sistemas usam poderosos algoritmos de convolução e superposição assim como algoritmos de Monte 
Carlo $^{4}$ para o cálculo da dose administrada ao paciente num tratamento com radioterapia. Para que o cálculo das doses realizado pelo TPS seja acurado é fundamental o conhecimento do espectro de fluência de fótons de raios $X$ que entrega a máquina de tratamento, o acelerador linear $^{1-4}$. Neste trabalho foi desenvolvido um método indireto para reconstruir o espectro de fluência de fótons de aceleradores lineares de uso clinico a partir da medição das curvas de transmissão através de blocos de alumínio, e do uso do método de recozimento simulado generalizado $^{12}$ (GSA, do inglês Generalized Simulated Annealing); os resultados mostram um grande acordo entre o espectro simulado e o espectro obtido do TPS XiO 4.62.00. Para a validação do espectro reconstruído calculamos as grandezas dosimétricas associadas como a porcentagem de dose em profundidade (PDD, do inglês Percentage Depth Dose) e o índice de qualidade do feixe $\mathrm{TPR}_{20 / 10}$.

\section{Materiais e Métodos}

Foram medidos os dados das curvas de transmissão $^{1-5}$ para fótons no acelerador linear SIEMENS ONCOR com energia nominal de $6 \mathrm{MV}$ através de blocos de alumínio. As curvas de transmissão foram medidas com uma câmara de ionização FARMER FC65-G, conectada a um eletrômetro IBA-DOSE1, calibrada e que é utilizada em medidas de controle de qualidade no Serviço de Radioterapia do Hospital Das Clinicas da Faculdade de Medicina de Ribeirão Preto, registrando como leitura do eletrômetro a carga produzida na câmara de ionização em unidades de nano Coulomb ( $\mathrm{nC}$ ) depois de que o feixe transmitido passa através de diferentes espessuras do material atenuador de acordo com o esquema dado na Figura 1.

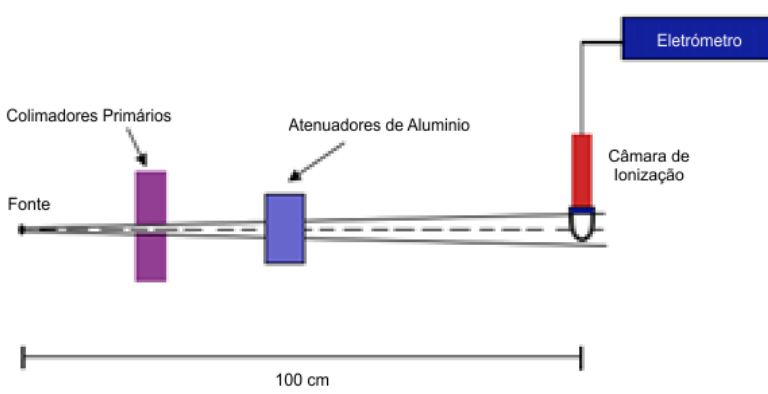

Figura 1. Esquema de medição das Curvas de Transmissão.

Depois de obtidas as curvas de transmissão, as medidas de transmissão foram normalizadas é inseridas num arquivo para ser processado pelo nosso programa GSA-1.0 escrito em $\mathrm{C}++$, o qual realiza o ajuste da transmissão medida, e a transmissão calculada, e faz a minimização da função de custo previamente construída, para assim obter o espectro de fluência de fótons usando 0 algoritmo de $\mathrm{GSA}^{8,12}$. Foram feitos diferentes testes com o algoritmo construído para sua validação previa mediante a minimização de funções previamente conhecidas e logo depois foi implementado este algoritmo na reconstrução de espectros de nosso interesse.

\subsection{Fluência de Fótons}

A fluência de fótons $^{1-4}$ é uma grandeza fundamental a partir da qual todas as grandezas importantes relativas ao feixe de radiação podem ser calculadas; o cálculo da fluência pode ser feito a partir de medições de transmissão ${ }^{8}$ tornando-se um problema de caráter inverso que pode ser resolvido usando 0 método de $\mathrm{GSA}^{8,12}$. Consideremos um feixe de raios $X$ com distribuição de fluência $\Phi_{E}(E)$ que depois de atravessar um certo número $n$ de atenuadores com espessuras $t_{1}$, $t_{2}, . ., t_{j}, . ., t_{n}$ e densidades $\rho_{1}, \rho_{2}, . . \rho_{j}, . ., \rho_{n}$ de um material tem a fluência $\Phi_{E}^{\prime}(E)$ na equação (1):

$$
=\emptyset_{E}(E) \exp \left[-(\mu / \rho) \sum_{j=0}^{n} \rho_{j} t_{j}\right]
$$

em que $(\mu / \rho)$ é o coeficiente de atenuação o qual depende da energia ${ }^{9}$.

\subsection{Generalized Simulated Annealing}

O GSA ${ }^{8,12}$ é um método estocástico que permite encontrar o mínimo de função de custo $f\left(\Phi_{\text {trial }}\right)$ como a equação (2), mediante o resfriamento controlado da temperatura fazendo um ciclo iterativo baixando a temperatura (esquema de esfriamento) até o sistema alcançar seu estado cristalino de mínima energia. O método consiste em supor uma solução inicial para a fluência $\Phi_{\text {trial }}$ e encontrar novas soluções validadas pelo critério de Metropolis $^{7,10}$.

$$
\begin{aligned}
f\left(\emptyset_{\text {trial }}\right)= & \frac{1}{m} \sum_{n=0}^{m-1}\left[T^{(n)}-S^{(n)}\right]^{2} \\
& +\frac{\lambda}{k-2} \sum_{s=1}^{k-1} \mid \hat{\phi}_{\text {trial }}^{(s)} \\
& -\hat{\phi}_{\text {trial }}^{(s-1)} \mid
\end{aligned}
$$

$\widehat{\emptyset}_{\text {trial é uma fluência de ensaio normalizada }}{ }^{7}$ obtida a partir da divisão de cada um dos elementos de $\Phi_{\text {trial }}$ pelo máximo calculado $\max \left(\Phi_{\text {trial }}\right), T^{(n)}$ é a transmissão calculada ${ }^{8}, S^{(n)}$ é a transmissão medida ${ }^{8}, \lambda$ é um parâmetro de regularização, $m$ é o número de medidas feitas de transmissão, $k$ é o número de canais de energia para a reconstrução do espectro. A temperatura controla o processo de recozimento e para nosso caso desce de acordo a um esquema de esfriamento geométrico governado pelo tempo $t$, dado por $T_{a}{ }^{(\mathrm{t})}=\alpha^{t} T_{a}{ }^{(0)}$, em que $T_{a}{ }^{(0)}$ é uma temperatura inicial do sistema e $\alpha \in(0,1)$ e a taxa de esfriamento, existindo outro esquema para uma 
temperatura visitante $T_{v}^{\left({ }^{(t)}\right.}$ da equação (3) em que $\ln _{\breve{\boldsymbol{q}}_{v}}$ é a função logaritmo generalizado ${ }^{6}$ :

$$
T_{v}^{(t)}=T_{v}^{(0)} \frac{\ln _{\breve{q}_{v}}(2)}{\ln _{\breve{q}_{v}}(t+1)}
$$

\section{Resultados}

$\mathrm{Na}$ Tabela 1 observa-se as leituras da câmara de ionização em unidades de carga elétrica [nC] e sua correspondente normalização. Na Tabela 2 observa-se os parâmetros usados pelo programa GSA-1.0 para reconstruir os espectros: número de canais $k$, número de iterações máximo $t_{\max }$, número de soluções de ensaio em cada iteração $n_{r}$, o valor do parâmetro de generalização $q_{v}$, o parâmetro de regularização $\lambda$ e o coeficiente da taxa de esfriamento $\alpha$ em que também temos resumido o cálculo da energia média para o espectro obtido dando um resultado de $2,05 \mathrm{MeV}$ que é um valor muito próximo a energia média obtida do espectro do TPS que dá um valor de 2,075 MeV.

Tabela 1. Dados medidas Transmissão no Acelerador de $6 \mathrm{MV}$.

\begin{tabular}{cccccc}
\hline $\begin{array}{c}\text { Espess. } \\
\text { de Al }\end{array}$ & Leitura & Transm. & $\begin{array}{c}\text { Espess. } \\
\text { de Al }\end{array}$ & Leitura & Transm. \\
\hline$(\mathrm{cm})$ & $(\mathrm{nC})$ & (relativa) & $(\mathrm{cm})$ & $(\mathrm{nC})$ & (relativa) \\
\hline 0,0 & 16,9 & 1 & 10 & 5,62 & 0,3329 \\
0,1 & 16,7 & 0,9876 & 15 & 3,38 & 0,2002 \\
0,2 & 16,5 & 0,9751 & 16 & 3,06 & 0,1813 \\
0,3 & 16,3 & 0,9639 & 20 & 2,08 & 0,1233 \\
1,0 & 15,0 & 0,8886 & 21 & 1,89 & 0,1121 \\
1,1 & 14,8 & 0,8786 & 25 & 1,29 & 0,0761 \\
1,2 & 14,7 & 0,8685 & 30 & 0,818 & 0,0485 \\
1,3 & 14,5 & 0,8590 & 35 & 0,523 & 0,0310 \\
5,0 & 9,56 & 0,5662 & 40 & 0,341 & 0,0202 \\
6,0 & 8,57 & 0,5076 & 45 & 0,224 & 0,0133 \\
\hline
\end{tabular}

Tabela 2 - Parâmetros para reconstrução do Espectro de 6 MV.

\begin{tabular}{lcc}
\hline Item & Representação & Valor \\
\hline Número de canais & $\mathrm{k}$ & 33 \\
Numero de iterações & $t_{\max }$ & 500 \\
Número de soluções de ensaio & $n_{r}$ & 200 \\
Parâmetro de generalização & $q_{\mathrm{v}}$ & 0,8 \\
Parâmetro de regularização & - & 2000 \\
Coeficiente de esfriamento & $\alpha$ & 0,9 \\
\hline Energia média $(\mathrm{MeV})$ & \multicolumn{2}{c}{2,05} \\
\hline
\end{tabular}

Na Figura 2 mostra-se o espectro de fluência de fótons reconstruído com a técnica $\mathrm{GSA}^{10}$ obtida com o programa GSA-1.0 (linha com pontos azuis),

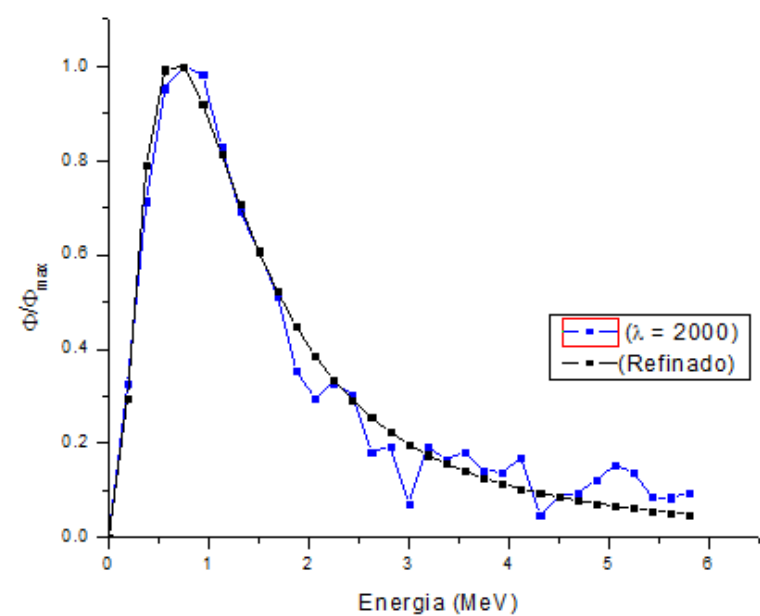

Figura 2. Espectro de fluência de fótons de $6 \mathrm{MV}$ refinado (linha com pontos pretos) e o espectro normalizado (linha com pontos azuis) obtido pela técnica de GSA para alumínio em função da energia $\mathrm{E}$ do fóton.

e o espectro refinado (linha com pontos pretos), onde pela extrapolação da curva do espectro refinado obteve-se a $E_{\max }=7,87 \mathrm{MeV}$.

Na Figura 3, fazemos uma comparação entre o espectro de fluência de fótons de $6 \mathrm{MV}$ obtido pelo programa GSA $-1.0^{10}$, e o espectro obtido do sistema de planejamento XiO 4.62.00, onde observa-se uma pequena diferença na região de 3 a $5 \mathrm{MeV}$, entre o espectro do TPS (pontos pretos) e o espectro reconstruído pelo programa GSA-1.0 (linha verde com pontos azuis), mas além disso, como se observa, existe um bom acordo entre o espectro calculado pelo uso da técnica de GSA, e o espectro obtido do TPS, a divergência se deve ao método de reconstrução usado.

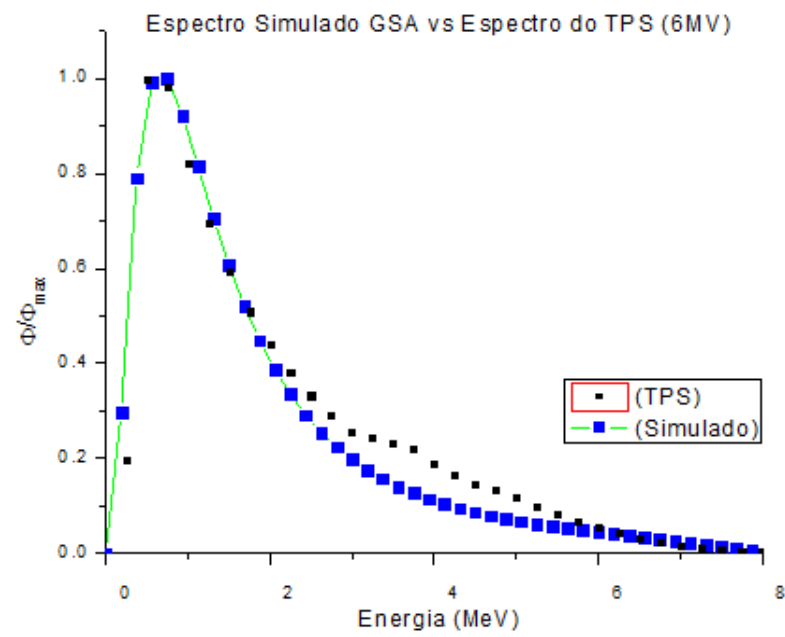

Figura 3. Comparação do espectro de fluência de fótons de $6 \mathrm{MV}$ obtido pela técnica de GSA e o espectro do sistema de planejamento $\mathrm{XiO}$ 4.62.00.

\subsection{Cálculos de PDD e TPR $20 / 10$}

Com o propósito de validar os espectros reconstruídos pela técnica GSA ${ }^{10}$ obteve-se a PDD usando o código Monte Carlo PENELOPE ${ }^{11}$, desenhando uma geometria adequada com um objeto simulador de água de dimensões $40 \times 40 \times$ $51 \mathrm{~cm}^{3}$, e colocando uma fonte a $100 \mathrm{~cm}$ de distancia (SSD, do inglês source surface distance) considerando uma fonte espectral com o espectro 
calculado pelo método GSA anterior. O resultado para a PDD calculada com o código PENELOPE é mostrado na Figura 4.

$\mathrm{Na}$ Figura 4 se pode observar que existe um bom acordo entre a PDD simulada por PENELOPE e a PDD medida para o acelerador linear em condições de referência.

Para o cálculo do índice de qualidade do feixe ${ }^{3}$ $\mathrm{TPR}_{20 / 10}$ usamos a $\mathrm{PDD}_{20 / 10}$, calculada a partir da PDD reconstruída, e a fórmula dada no IAEA-TRS $398^{3}$ a partir da equação:

$$
\begin{aligned}
\operatorname{TPR}_{20} /_{10} & =1,2661 P D D_{20} / 10 \\
& -0,0595
\end{aligned}
$$

Em que a $\mathrm{PDD}_{20 / 10}$ é definida como o quociente das porcentagens de doses em profundidade para as profundezas de $20 \mathrm{~cm}$ e $10 \mathrm{~cm}$ para um tamanho de campo de $10 \mathrm{~cm} \times 10 \mathrm{~cm}$, definido na superfície do objeto simulador de água a uma SSD de $100 \mathrm{~cm}$. O cálculo da TPR $20 / 10$ para a energia de $6 \mathrm{MV}$ é dado na Tabela 3.

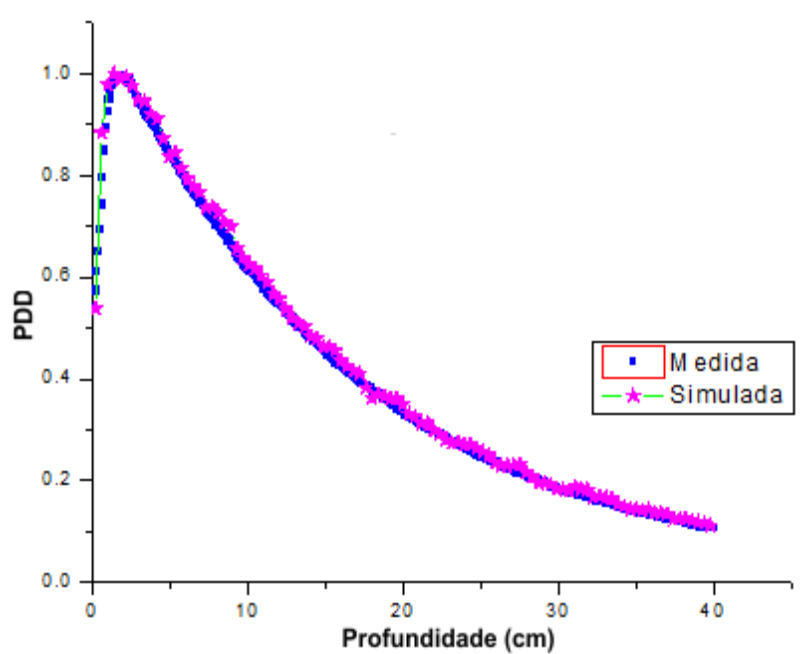

Figura 4. Curvas de porcentagem de dose em profundidade simulada por PENELOPE e medida para o acelerador linear em condições de referência.

Este resultado foi comparado com os dados de $\left[\mathrm{TPR}_{20 / 10}\right]^{\text {ref }}$ usados no serviço de radioterapia do Hospital das Clinicas da Faculdade de Medicina da Universidade de São Paulo de Ribeirão Preto observando-se um bom acordo com o resultado obtido num erro de calculo menor a $1 \%$.

Tabela 3 - Cálculo do TPR $20 / 10$ para fótons de $6 \mathrm{MV}$.

\begin{tabular}{ccc}
\hline $\mathrm{PDD}_{20 / 10}$ & $\mathrm{TPR}_{20 / 10}$ & {$\left[\mathrm{TPR}_{20 / 10}\right]^{\text {ref }}$} \\
\hline 0,578 & 0,672 & 0,673 \\
\hline$\%$ de Erro & \multicolumn{3}{c}{$<1 \%$} \\
\hline
\end{tabular}

\section{Discussão}

O cálculo do espectro de fótons do acelerador lineal de 6MV mostra um grande acordo com o espectro obtido do TPS, isso permite usar esse espectro para obter a porcentagem de dose profunda PDD a partir da qual obtemos o índice de qualidade do feixe $\mathrm{TPR}_{20 / 10}$. Estes cálculos obtidos neste trabalho a partir do espectro de fluência de fótons reconstruído indicam que o método de GSA é uma potente ferramenta para a minimização de funções de custo que pode ser usada na reconstrução de espectros de aceleradores lineares clínicos, e para calcular quantidades de interesse como a energia média do espectro do feixe de fótons que são difíceis de medir diretamente na rotina clinica.

\section{Conclusões}

O método de GSA é uma potente ferramenta pra reconstrução de espectros de aceleradores lineares de uso clinico, não requer um conhecimento rigoroso da geometria e os materiais do cabeçote do acelerador que são necessários para implementar uma simulação Monte Carlo, já que usa um método inverso com base nos dados de transmissão o qual torna mais accessível para o cálculo de grandezas de interesse relativas ao feixe clínico com grande acurácia, como a energia media, PDDs, e o índice de qualidade do feixe $\mathrm{TPR}_{20 / 10}$.

\section{Agradecimentos}

Agradeço à Coordenação de Aperfeiçoamento de Pessoal de Nível Superior CAPES pela ajuda para a realização deste trabalho e ao Serviço de Radioterapia do Hospital das Clinicas Faculdade de Medicina de Ribeirão Preto que tornou possível as medições apresentadas.

\section{Referências}

1. Attix FH. Introduction to Radiological Physics and Radiation Dosimetry. New York: John Wiley \& Sons; 1986.

2. ICRU: Prescribing, recording, and reporting photon beam therapy (supplement to ICRU report 50). In ICRU Report Volume 62. Bethesda: International Commission on Radiation Units and Measurements; 1999.

3. IAEA: Absorbed dose determination in external beam radiotherapy: an international code of practice for dosimetry based on standards of absorbed dose to water, Technical Report Series No. 398. Vienna: IAEA, 2000.

4. KHAN, FM. The Physics of Radiation Therapy. Philadelphia, USA: Lippncott Williams \& Wilkins; 2003.

5. Knoll GF. Radiation Detection and Measurement. 4. Ed. Hoboken, NJ: John Wiley \& Sons; 2010.

6. Martínez AS, González RS, Terçariol CAS. Generalized probability functions. Adv. Math. Phys. 2009; 206176.

7. Menin $\mathrm{OH}$, Martinez AS, Costa AM. Reconstruction of bremsstrahlung spectra from attenuation data using generalized simulated annealing. Applied Radiation and Isotopes. 2016; 111:80-85.

8. Nisbet A, Weattherburn H, Fewick JD, Mcvey G. Spectral reconstruction of clinical megavoltage photon beams and the implications of spectral determination on the dosimetry of such beams. Physics in Medicine and Biology 1998; 43:1507-1521.

9. National Institute of Standards and Technology-NIST. XCOM: Photon Cross Sections Database: http://www.nist.gov/physlab/data/xcom/index.cfm; 2013.

10. Manrique, JPO. Reconstrução do espectro de fótons de aceleradores lineares clínicos com base na curva de transmissão e no algoritmo de recozimento simulado generalizado [Dissertação de Mestrado]. Ribeirão Preto: Universidade de São Paulo, Faculdade de Filosofia, Ciências e Letras de Ribeirão Preto; 2015 [citado 2017-0419]. Disponível em: http://www.teses.usp.br/teses/disponiveis/59/59135/tde31012016-151001/. 
11. Salvat F, Fernández-Varea JM, Sempau J. PENELOPE2008: A Code System for Monte Carlo Simulation of Electron and Photon Transport; OECD/NEA Data Bank: Issy-les-Moulineaux, France, 208.

12. Tsallis C, Stariolo DA. Generalized simulated annealing. Physics A 1996; 233(1):395-406,

\section{Contato:}

John Peter Oyardo Manrique

Alessandro Martins Da Costa

Departamento de Física - FFCLRP - USP

Av. Bandeirantes, 3900

Vila Monte Alegre

Ribeirão Preto - SP - Brasil 14040-901

Fone: +55-16-3315-4670

Fax: +55-16-3315-4887

johnp067@usp.br, amcosta@usp.br. 